\title{
Indocyanine green angiography findings in patients with long-standing Vogt-Koyanagi- Harada disease: a cross-sectional study
}

Felipe T da Silva' ${ }^{1}$ Carlos E Hirata', Viviane M Sakata ${ }^{1}$, Edilberto Olivalves ${ }^{1}$, Rony Preti ${ }^{2}$, Sergio LG Pimentel ${ }^{2}$, Andre Gomes ${ }^{2}$, Walter Y Takahashi ${ }^{2}$, Rogerio A Costa ${ }^{3}$ and Joyce H Yamamoto ${ }^{1 *}$

\begin{abstract}
Background: To investigate indocyanine green angiography (ICGA) findings in patients with long-standing Vogt-Koyanagi-Harada (VKH) disease and their correlation with disease activity on clinical examination as well as with systemic corticosteroid therapy.

Methods: Twenty-eight patients (51 eyes) with long-standing ( $\geq 6$ months from disease onset) VKH disease whose treatment was tapered based only in clinical features were prospectively included at a single center in Brazil. All patients underwent standardized clinical evaluation, which included fundus photography, fluorescein angiography and ICGA. Clinical disease activity was determined based in the Standardization in Uveitis Nomenclature Working Group. Fisher exact test and logistic regression models were used for statistical analysis.

Results: Disease-related choroidal inflammation on ICGA was observed in 72.5\% (31 of 51 eyes). Angiographic findings suggestive of (choroidal and/or retinal) disease activity were not observed on FA. Clinically active disease based on clinical evaluation was observed in $41.2 \%$ (21 of 51 eyes). In these 21 eyes, disease-related choroidal inflammation on ICGA was observed in 76.2\% (16 of 21 eyes); in the remaining eyes (without clinical active disease) disease-related choroidal inflammation on ICGA was observed in $70.0 \%$ (21 of 30 eyes). In respect to systemic corticosteroid therapy, 10 patients (18 of 51 eyes) were under treatment with prednisone. In these 10 (18 of 51 eyes) patients, disease-related choroidal inflammation on ICGA was observed in 83.3\% (15 of 18 eyes); in the remaining patients (33 of 51 eyes) disease-related choroidal inflammation on ICGA was observed in 66.7\% (22 of 33 eyes).

Conclusion: ICGA findings suggestive of disease-related choroidal inflammation were observed in a considerable proportion of patients with long-standing VKH disease, independent of the inflammatory status of the disease on clinical examination or current use of systemic corticosteroid. Therefore, the current study reinforces the crucial role of ICGA to assist the management and treatment of patients with long-standing VKH disease.
\end{abstract}

Keywords: Angiography, Indocyanine green, Choroid, Diagnosis, Inflammation, Vogt-Koyanagi-Harada

\footnotetext{
* Correspondence: joycehy@uol.com.br

${ }^{1}$ Uveitis Service, Department of Ophthalmology, Hospital das Clínicas,

Universidade de São Paulo, São Paulo, SP, Brazil

Full list of author information is available at the end of the article
} 


\section{Background}

Vogt-Koyanagi-Harada (VKH) disease is a T-cell altered immune process that is directed at the melanocytes [1]. The diagnosis is essentially based on the observation of bilateral diffuse choroiditis of acute onset following flulike prodromic symptoms, and prompt treatment with systemic corticosteroid and/or immunosuppressant has been recommended. In spite of aggressive treatment during the acute phase of the disease, slowly progressive fundus depigmentation has been observed over time [2], even in the absence of detectable clinical signs of disease activity. There is increasing evidence that this slowly progressive fundus depigmentation is related to some disease activity undetected on regular clinical examination and is due to insufficient therapy; it does most probably not occur as part of the natural history of $\mathrm{VKH}$ disease [3].

In this sense, indocyanine green angiography (ICGA) may be an interesting ancillary tool. As the dye fluoresces in the near infrared wavelength, indocyanine green is particularly useful for imaging of the choroidal vascular compartment. In fact, the role of ICGA in detecting choroidal inflammation in patients with $\mathrm{VKH}$ disease has been investigated [4-7], mainly in European and Asian countries. Its relevance has been pointed out to either confirm elements already revealed by clinical examination or to determine the inflammatory status of the fundus and monitor $\mathrm{VKH}$ disease activity during follow-up, thus assisting the treatment process [4-7].

The use of ICGA for monitoring VKH disease activity, however, is not universally accepted $[8,9]$. Moreover, some of the ICGA findings suggestive of choroidal inflammation related to the disease may share angiographic features with those findings observed in patients under systemic corticosteroid treatment [10-13]. In the current report, we studied the ICGA findings in patients with long-standing $\mathrm{VKH}$ disease (i.e., patients who had more than 6 months from disease onset, regardless of the disease activity status determined on clinical examination). The possible correlation between ICGA findings and clinical disease activity as well as systemic corticosteroid therapy was investigated.

\section{Methods}

\section{Subjects}

Patients with long-standing VKH disease were prospectively enrolled in the study from March 2007 through October 2008 at a single center in Brazil (Uveitis Service, Hospital das Clínicas, Universidade de São Paulo, São Paulo, SP, Brazil) after written informed consent was obtained.

The diagnosis of VKH disease was based on the Revised Diagnostic Criteria proposed by the International Committee on Nomenclature [14]. Patients who were eligible for inclusion in the study had been treated during the acute phase with oral prednisone administered at $1.0-1.5 \mathrm{mg} / \mathrm{kg}$ daily, which was tapered according to disease activity over a period of time of at least 6 months. During this period disease activity was determined based on clinical examination and on fluorescein angiography (FA) findings.

The characterization of long-standing disease was based in the presence of a minimum time interval of 6 months from the onset of VKH disease, independently of the current clinical [phase] status of the disease. Patients with known allergy to fluorescein and/or indocyanine green dyes and media opacities that could prevent adequate fundus imaging were not eligible for study participation.

During the study period, disease activity was determined based on clinical evaluation, in accordance to the Standardization of Uveitis Nomenclature (SUN) Working Group [15]. Those eyes presenting cells in the anterior or posterior chamber or disc hyperemia, as well as any sign suggestive of posterior inflammation (for example, optic disc hyperfluorescence) on FA, were considered to have clinically active disease. Patients with clinically active disease by the time of initial study evaluation were treated with topical corticosteroid, associated or not with oral prednisone and/or immunosuppressant.

\section{Fundus photography and angiographic studies}

Eligible patients underwent fundus photography (color and red-free) as well as angiographic studies (fluorescein and indocyanine green) using a conventional fundus camera system (TRC-50IX/IMAGEnet; Topcon Inc., Tokyo, Japan). Angiographic studies followed a standardized protocol previously described for the investigation of inflammatory eye diseases [16], and the eye with more pronounced fundus changes on clinical examination was selected for documentation of the initial transit phase of the dyes.

The ICGA data were evaluated by a retina specialist in a masked fashion. The following ICGA findings were categorically analyzed: 1) diffusely leaking choroidal vessels in the intermediate phase ("fuzzy vessels"), 2) diffuse choroidal hyperfluorescence in the late phase, and 3) hypofluorescent dark dots in the intermediate phase with later isofluorescence $[6,7,16]$. Those eyes presenting at least 2 of these findings were considered to have disease-related choroidal inflammation on ICGA.

\section{Ethics}

This study protocol followed the statements of the Declaration of Helsinki and was approved by the Ethics in Research Committee analyzing research projectsCAPPesq from the Clinical Board of the Medical School 
of the University of São Paulo, under protocol \#10121/ 2008.

\section{Statistical analysis}

The correlation between the ICGA findings (i.e., diseaserelated choroidal inflammation) and disease activity on clinical examination (i.e., clinical activity) at the time of the angiographic study was evaluated using Fisher exact test. Logistic regression models were used to analyze the relationship between disease-related choroidal inflammation on ICGA and clinical activity as well as the use of systemic corticosteroid therapy. Data analysis and statistical tests were done using SPSS 15.0 (SPSS Science, Chicago, Illinois, USA) statistical software. The significance level was set at 0.05 for all tests.

\section{Results}

Twenty-eight patients with long-standing VKH disease (51 eyes) were included in the study (in 5 patients, one eye was excluded due to media opacities). The ICGA findings were correlated with clinical activity and systemic corticosteroid therapy. The baseline characteristics are presented in Table 1.

At least one individual ICGA feature was observed in patients with long-standing VKH disease. The fuzzy vessels were observed in $80.4 \%$ ( 41 of 51 eyes), late diffuse hyperfluorescence in $78.4 \%$ ( 40 of 51 eyes), and dark dots in the intermediate phase with later isofluorescence in $19.6 \%$ (10 of 51 eyes). The presence of at least 2 of these ICGA features, thus characterizing disease-related choroidal inflammation on ICGA, was observed in $72.5 \%$ (37 of 51 eyes) (Figure 1). On FA, all eyes exhibited areas of hypofluorescence due to blockage interspersed with areas of hyperfluorescence due to window defects. Late hyperfluorescence due to staining of subretinal fibrovascular tissue was also observed in 37.3\% (19 of 51 eyes). FA findings suggestive of (choroidal and/ or retinal) disease activity were not observed.

In respect to clinical activity, $41.2 \%$ (21 of 51 eyes) were considered to have clinically active disease based on clinical evaluation. In all 21 eyes clinical signs indicative of some degree of anterior chamber inflammation (cells + to +++ ) were observed on clinical examination. Clinical signs suggestive of inflammation of the posterior segment were not observed on clinical examination.

In respect to systemic corticosteroid therapy, 10 patients (18 of 51 eyes) were under treatment with prednisone (with or without immunosuppressant agents). Three patients ( 5 of 51 eyes) were under treatment with immunosuppressant agents, and 15 patients (28 of 51 eyes) were using only topical treatment (Table 2).

The possible correlation of disease-related choroidal inflammation on ICGA with clinical activity and with systemic corticosteroid therapy was evaluated. In the 21
Table 1 Demographics and clinical characteristics of the patients with Vogt-Koyanagi-Harada and long-standing disease

\begin{tabular}{|c|c|c|}
\hline Description & & \\
\hline Number of patients (eyes) & 28 & (51) \\
\hline Age (years), mean ( \pm 1SD) & 39.9 & \pm 13.0 \\
\hline Gender, male (\%) & 4 & $(14.3)$ \\
\hline \multicolumn{3}{|l|}{ Race, n (\%) } \\
\hline White & 12 & $(42.8)$ \\
\hline Mestizo & 10 & $(35.7)$ \\
\hline Black & 4 & $(14.3)$ \\
\hline Asian/Oriental & 2 & $(7.1)$ \\
\hline \multicolumn{3}{|l|}{ Revised Diagnostic Criteria category [14], n (\%) } \\
\hline Complete & 7 & $(25.0)$ \\
\hline Incomplete & 14 & $(50.0)$ \\
\hline Probable & 7 & $(25.0)$ \\
\hline \multicolumn{3}{|l|}{ Clinical activity (SUN) [15], n (\%) } \\
\hline Remission & 11 & $(39.3)$ \\
\hline Inactive ${ }^{\S}$ & 5 & $(17.8)$ \\
\hline Active & 12 & $(42.8)$ \\
\hline \multicolumn{3}{|l|}{ Use of systemic medication, n (\%) } \\
\hline Prednisone only & 5 & $(17.8)$ \\
\hline Prednisone and immunosuppressant & 5 & $(17.8)$ \\
\hline Immunosuppressants only & 3 & $(10.7)$ \\
\hline None & 15 & $(53.6)$ \\
\hline Disease duration (in months), median (range) & 91.5 & $(9-348)$ \\
\hline
\end{tabular}

Inactive disease for $\geq 3$ months after discontinuing all treatments for eye disease.

${ }^{\S}$ Grade 0 cells.

eyes with clinically active disease based on clinical evaluation disease-related choroidal inflammation on ICGA was observed in $76.2 \%$ (16 of 21 eyes). In the remaining 30 eyes (without clinical active disease) disease-related choroidal inflammation on ICGA was observed in $70.0 \%$ (21 of 30 eyes). In the patients (18 of 51 eyes) under systemic corticosteroid therapy, disease-related choroidal inflammation on ICGA was observed in $83.3 \%$ (15 of 18 eyes); in the remaining patients (33 of 51 eyes) diseaserelated choroidal inflammation on ICGA was observed in $66.7 \%$ ( 22 of 33 eyes).

\section{Discussion}

The current study demonstrated that ICGA findings suggestive of disease-related choroidal inflammation was observed in a considerable proportion of patients with long-standing VKH disease whose treatment was tapered according to clinical and FA evaluation. Disease-related choroidal inflammation on ICGA was highly prevalent even among patients under systemic treatment as well as among those with no clinically detectable disease 


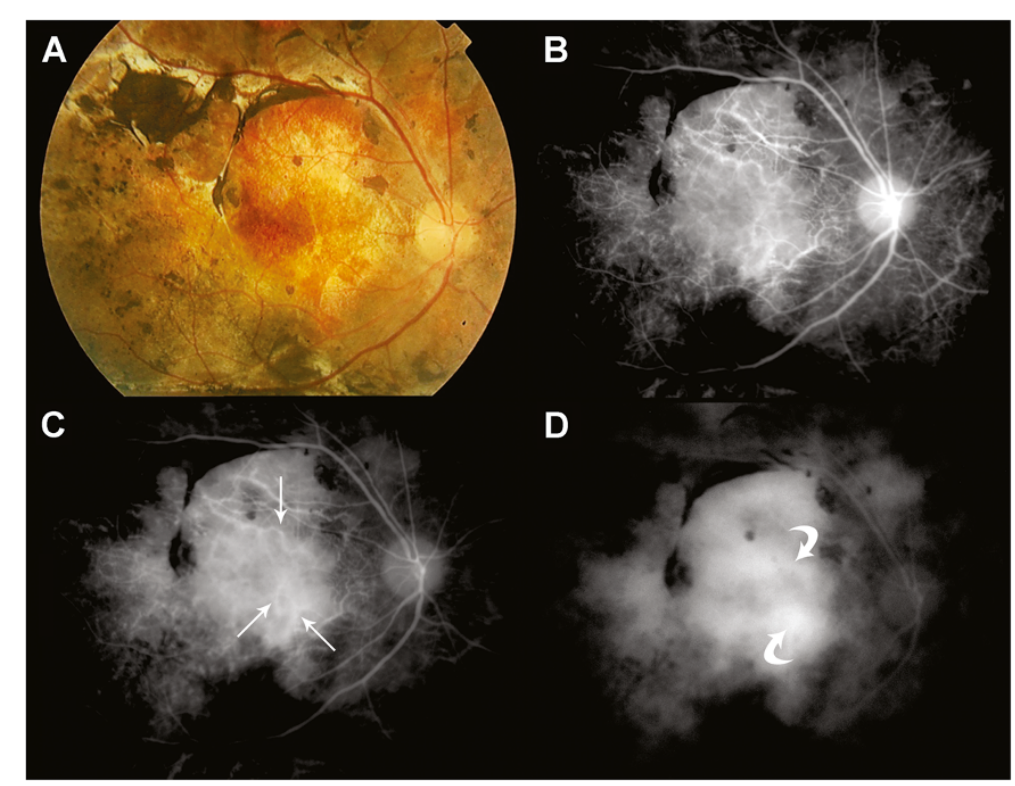

Figure 1 Color fundus photography $(A)$ as well as early, mid and late phase indocyanine green angiography (B,C,D, respectively) from a representative patient with Vogt-Koyanagi-Harada and long-standing disease. Note "fuzzy vessels" (arrows) on early and mid phases ICGA, and "late diffuse hyperfluorescence" (curved arrows) on late phase of the exam.

activity, thus confirming previous studies in European and Asian population and reinforcing the usefulness of ICGA to assist monitoring disease activity to better tailor treatment strategies.

Bouchenaki and Herbort described ICGA findings suggestive of choroidal involvement in acute VKH disease, which were less evident in the chronically evolving disease, and proposed ICGA as a useful tool to monitor the effect of steroid therapy [7]. Subtle ICGA findings in patients with VKH disease were subsequently shown to represent otherwise unnoticeable posterior segment disease-related activity [4-6]. These findings were

Table 2 Correlation between disease-related choroidal inflammation on indocyanine green angiography, clinical disease activity and systemic corticosteroid therapy in $\mathbf{2 8}$ patients (51 eyes) with Vogt-Koyanagi-Harada and longstanding disease

\begin{tabular}{|c|c|c|c|}
\hline & $\mathrm{N}$ (eyes) & $\begin{array}{c}\text { Disease-related choroidal } \\
\text { inflammation on ICGA, } n(\%)\end{array}$ & $p$ \\
\hline Total number of eyes & 51 & $37(72.5)$ & \\
\hline \multicolumn{4}{|c|}{ Anterior chamber inflammation (cells) } \\
\hline Yes & 21 & $16(76.2)$ & $0.626^{\dagger}$ \\
\hline No & 30 & $21(70.0)$ & \\
\hline \multicolumn{4}{|c|}{ Systemic treatment at the time of ICGA } \\
\hline Prednisone & 18 & $15(83.3)$ & $0.326^{\dagger}$ \\
\hline Immunosuppressant & 5 & $3(60.0)$ & \\
\hline No & 28 & 19 (67.8) & \\
\hline
\end{tabular}

${ }^{\dagger}$ Results of Fisher's exact test. described in the context of $\mathrm{VKH}$ disease during anterior segment relapses in the chronic phase and in patients tapering treatment in the convalescent phase of the disease. In both scenarios, intensification of immunosuppressive treatment resolved both, clinically apparent as well as the underlying disease process. Kawaguchi et al. coined the term partially treated VKH disease to describe patients in the convalescent phase whose treatment was intense enough to abolish anterior segment activity while leaving signs of choroidal involvement on ICGA, and who experienced progressive fundus depigmentation (sunset-glow fundus) [4]. In the current study, roughly three quarters of the eyes with long-standing $\mathrm{VKH}$ disease presented ICGA findings suggestive of disease-related choroidal inflammation regardless of the presence of activity on clinical examination. In fact, 70.0\% of eyes demonstrated disease-related choroidal inflammation on ICGA without clinical evidence of disease activity, thus suggesting the presence of subclinical choroidal inflammation. Considering treatment regimen by the time of disease diagnosis, the majority of the patients included in this study had received early high-dose treatment. However, they were not systematically monitored with ICGA during the systemic treatment course, and clinical appraisal and fluorescein angiography were the only parameters used to taper treatment. Coupled with previous reports of better outcomes in more intensely treated patients, these observations provide additional evidence to support the possible role of ICGA in the adjustment of therapy at this stage of VKH disease [4,5,14-17]. The 


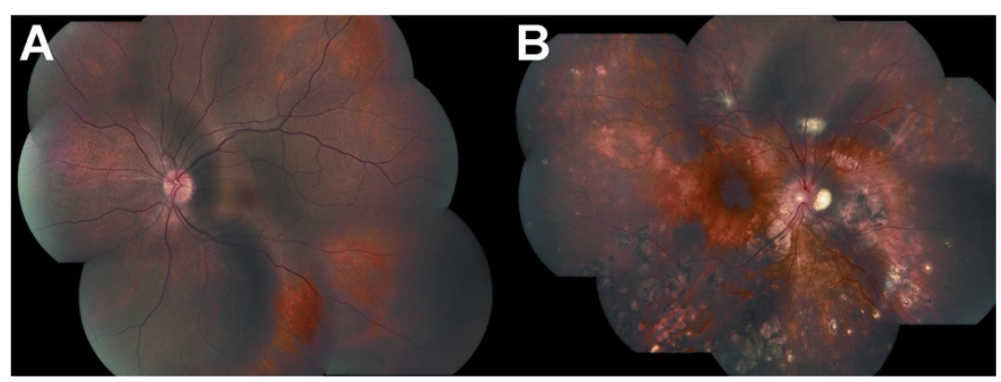

Figure 2 Color fundus photography (photomontage) from patients with Vogt-Koyanagi-Harada and long-standing disease. Mild (A) and severe disease (B) according to fundus-based disease severity grading (as per standardized analytic framework for ocular fundus alterations $[22,23])$.

importance of ICGA guided management of VKH disease to meaningfully assess choroidal inflammation has been recently reinforced by Bouchenaki and Herbort [3]. These authors proposed that zero tolerance to subclinical choroidal inflammation could avoid irremediable evolution towards sunset glow fundus [3].

In the current study, fuzzy vessels and late diffuse hyperfluorescence were the most frequently findings observed on ICGA, thus suggesting some alteration in choroidal vessels' permeability and choriocapillaris involvement [6]. Dark dots were considered only if they became isofluorescent (or mildly hyperfluorescent) in late-phase frames [6], and were the least observed ICGA finding in the current study. These observations are in line with previous histopathologic studies in patients with VKH and chronic disease in which a diffuse, nongranulomatous choroiditis accompanied by choriocapillaris involvement was described [1]. Additional findings identifiable on angiographic studies that have been correlated to serious ocular manifestations and/or severe choroidal inflammation at the acute uveitic stage of VKH disease [18-21], such as the presence of choroidal folds as reported by Wu et al.. [18], were not identified in the current study.

Disease-related choroidal inflammation on ICGA was apparently more easily observed among those patients with milder fundus changes than in those with more severe fundus changes in the current study (Figure 2; Additional file 1: Table S3) [22,23]. It is possible that some eyes with fundus-based severe disease could also present subclinical choroidal inflammation that remains undetected on ICGA due to a combination of factors, such as the limited ability to identify ICGA signs of choroidal inflammation in a severely altered fundus with destruction and scarring of a considerable proportion of the choroidal stroma [6]. Naturally, the identification of the ICGA findings indicative of choroidal inflammation in patients with mild fundus changes seems an easier task. It should be noted that this study has limitations due to the relatively small number of patients and its cross- sectional design. In addition, the misinterpretation of the ICGA findings should always be considered in studies of this nature. Future studies, combining ICGA with other fundus imaging modalities such as fundus autofluorescence and enhanced depth imaging optical coherence tomography may facilitate our understanding of the choroidal involvement in patients with VKH disease [24,25], independent of the severity of disease associated fundus alterations.

\section{Conclusions}

In the current study a considerable proportion of patients with long-standing VKH disease whose treatment was tapered based only on clinical and FA findings demonstrated ICGA findings suggestive of diseaserelated choroidal inflammation. Importantly, we have also demonstrated that these ICGA findings, which share some angiographic features with those associated with corticosteroid treatment, were observed independent of the use of systemic corticosteroid. Therefore, we herein provide additional evidence to support the use of ICGA for the detection of subclinical inflammation in patients with long-standing VKH disease, which may assist the management and treatment process of this entity, particularly in eyes with milder disease-related fundus changes.

\section{Additional file}

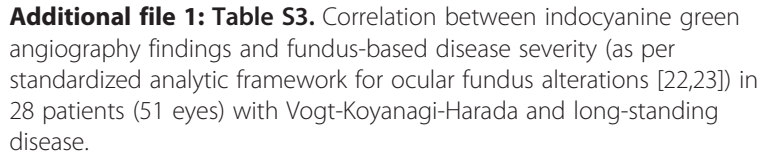

Additional file 1: Table S3. Correlation between indocyanine green angiography findings and fundus-based disease severity (as per standardized analytic framework for ocular fundus alterations [22,23]) in 28 patients (51 eyes) with Vogt-Koyanagi-Harada and long-standing disease.

\section{Competing interests}

The authors declare that they have no competing interests.

\section{Authors' contributions}

FTBGCS made substantial contribution to acquisition, analysis, interpretation of data, in drafting and revising the manuscript critically; CEH participated in the design, interpretation of data and in revising the manuscript critically; 
VMS and EO participated in revising the manuscript critically; RCP, SLGP, AVG participated in the analysis, interpretation of data and in revising the manuscript critically; WYT participated in the design, in the analysis, interpretation of data and in revising the manuscript critically; RAC participated in the analysis, interpretation of data, in drafting and revising the manuscript critically; JHY participated in the design, coordination, analysis, interpretation of data, in drafting and revising the manuscript critically. All authors read and approved the final manuscript.

\section{Acknowledgements}

This study was supported by scholarship 07/57154-9 and research grant 07/57155-5 from Fundação de Amparo à Pesquisa do Estado de São Paulo (FAPESP), São Paulo, SP, Brazil. The authors thank Professor Suel Abujamra for kindly allowing the indocyanine green angiography exams to be performed at his private practice, and Dr. Rogério Ruscitto do Prado, Statistics Division, Department of Preventive Medicine, Faculdade de Medicina da Universidade de São Paulo, São Paulo, SP, Brazil, for his assistance with statistical analysis.

\section{Author details}

'Uveitis Service, Department of Ophthalmology, Hospital das Clínicas, Universidade de São Paulo, São Paulo, SP, Brazil. 'Retina Section, Department of Ophthalmology, Hospital das Clínicas, Universidade de São Paulo, São Paulo, SP, Brazil. ${ }^{3}$ Division of Macula: Imaging \& Treatment, Centro Brasileiro de Ciências Visuais, Belo Horizonte, MG, Brazil. ${ }^{4}$ Rua Diana, 863 apto 91 J, São Paulo, SP 05019-000, Brazil.

Received: 19 March 2012 Accepted: 1 August 2012 Published: 13 August 2012

\section{References}

1. Rao NA: Pathology of Vogt-Koyanagi-Harada disease. Int Ophthalmol 2007, 27:81-85.

2. Suzuki S: Quantitative evaluation of "sunset glow" fundus in VogtKoyanagi-Harada disease. Jpn J Ophthalmol 1999, 43:327-333.

3. Bouchenaki N, Herbort CP: Indocyanine green angiography guided management of Vogt-Koyanagi-Harada disease. J Ophthalmic Vis Res 2011, 6:241-248.

4. Kawaguchi T, Horie S, Bouchenaki N, Ohno-Matsui K, Mochizuki M, Herbort CP: Suboptimal therapy controls clinically apparent disease but not subclinical progression of Vogt-Koyanagi-Harada disease. Int Ophthalmol 2010, 30:41-50.

5. Bacsal K, Wen DS, Chee SP: Concomitant choroidal inflammation during anterior segment recurrence in Vogt-Koyanagi-Harada disease. Am J Ophthalmol 2008, 145:480-486.

6. Herbort CP, Mantovani A, Bouchenaki N: Indocyanine green angiography in Vogt-Koyanagi-Harada disease: angiographic signs and utility in patient follow-up. Int Ophthalmol 2007, 27:173-182.

7. Bouchenaki N, Herbort CP: The contribution of indocyanine green angiography to the appraisal and management of Vogt-KoyanagiHarada disease. Ophthalmology 2001, 108:54-64

8. Stanga PE, Lim Jl: Hamilton P:Indocyanine green angiography in chorioretinal disease: indications and interpretation. An evidence-based update. Ophthalmology 2003, 110:15-24.

9. Yannuzzi LA: Indocyanine green angiography: a perspective on use in the clinical setting. Am J Ophthalmol 2011, 151:745-751.

10. Guyer DR, Yannuzzi LA, Slakter JS, Sorenson JA, Ho A, Orlock D: Digital indocyanine green videoangiography of central serous chorioretinopathy. Arch Ophthalmol 1994, 112:1057-1062.

11. Prünte C, Flammer J: Choroidal capillary and venous congestion in central serous chorioretinopathy. Am J Ophthalmol 1996, 121:26-34.

12. Lafaut BA, Salati C, Priem H, De Laey JJ: Indocyanine green angiography is of value for the diagnosis of chronic central serous chorioretinopathy in elderly patients. Graefes Arch Clin Exp Ophthalmol 1998, 236:513-521.

13. Kishi S, Yoshida O, Matsuoka R, Kojima Y: Serous retinal detachment in patients under systemic corticosteroid treatment. Jpn J Ophthalmol 2001, 45:640-647.

14. Read RW, Holland GN, Rao NA, et al: Revised diagnostic criteria for VogtKoyanagi-Harada disease: Report of an international comittee on nomenclature. Am J Ophthalmol 2001, 131:647-652.
15. Jabs DA, Nussenblatt RB, Rosenbaum JT: Standardization of uveitis nomenclature for reporting clinical data. Am J Ophthalmol 2005, 140:509-516.

16. Herbort CP, LeHoang P, Gueux-Croiser Y: Schematic interpretation of indocyanine green angiography in posterior uveitis using a standard protocol. Ophthalmology 1998, 105:432-440.

17. Read RW, Yu F, Accorinti M, et al: Evaluation of the effect on outcomes of the route of administration of corticosteroids in acute Vogt-KoyanagiHarada disease. Am J Ophthalmol 2006, 142:119-124.

18. Wu W, Wen F, Huang S, Luo G, Wu D: Choroidal folds in Vogt-KoyanagiHarada disease. Am J Ophthalmol 2007, 143:900-901.

19. Fardeau C, Tran TH, Gharbi B, Cassoux N, Bodaghi B, LeHoang P: Retinal fluorescein and indocyanine green angiography and optical coherence tomography in successive stages of Vogt-Koyanagi-Harada disease. Int Ophthalmol 2007, 27:163-172. Epub 2007 Feb 2.

20. Zhao C, Zhang M, Wen X, Dong F, Han B, Du H: Choroidal folds in acute Vogt-Koyanagi-Harada disease. Ocul Immunol Inflamm 2009, 17:282-288.

21. Tanigawa M, Ochiai H, Tsukahara Y, Ochiai Y, Yamanaka H: Choroidal folds in acute-stage vogt-koyanagi-harada disease patients with relatively short axial length. Case Report Ophthalmol 2012, 3:38-45. Epub 2012 Feb 1.

22. da Silva FT, Damico FM, Marin ML, et al: Revised diagnostic criteria for Vogt-Koyanagi-Harada disease: Considerations on the different disease categories. Am J Ophthalmol 2009, 147:339-345.

23. da Silva FT, Hirata CE, Olivalves E, Oyamada MK, Yamamoto JH: Fundusbased and electroretinographic strategies for stratification of late-stage Vogt-Koyanagi-Harada disease patients. Am J Ophthalmol 2009, 148:939-945.

24. Vasconcelos-Santos DV, Sohn EH, Sadda S, Rao NA: Retinal pigment epithelial changes in chronic Vogt-Koyanagi-Harada disease: fundus autofluorescence and spectral domain-optical coherence tomography findings. Retina 2010, 30:33-41.

25. Heussen FM, Vasconcelos-Santos DV, Pappuru RR, Walsh AC, Rao NA, Sadda SR: Ultra-wide-field green-light (532-nm) autofluorescence imaging in chronic Vogt-Koyanagi-Harada disease. Ophthalmic Surg Lasers Imaging 2011, 42:272-277.

doi:10.1186/1471-2415-12-40

Cite this article as: da Silva et al:: Indocyanine green angiography findings in patients with long-standing Vogt-Koyanagi-Harada disease: a cross-sectional study. BMC Ophthalmology 2012 12:40.

\section{Submit your next manuscript to BioMed Central and take full advantage of:}

- Convenient online submission

- Thorough peer review

- No space constraints or color figure charges

- Immediate publication on acceptance

- Inclusion in PubMed, CAS, Scopus and Google Scholar

- Research which is freely available for redistribution 